\title{
Cholinesterase as a biomarker to identify cases of pesticide poisoning
}

\author{
Colinesterasa como biomarcador para identificar casos de intoxicación por plaguicidas
}

\author{
Azucena Benítez-Medina ${ }^{a}$, Marco Antonio Ramírez-Vargas ${ }^{b}$
}

\begin{abstract}
:
The clinical care of the intoxicated, as well as the epidemiological surveillance activities of the population exposed to pesticides, require reference values to be able to make decisions based on science. Cholinesterase enzymes are biomarkers discovered in the early twentieth century that currently work as auxiliaries in the diagnosis of acute and chronic poisoning. Objective: To carry out a systematic review to identify the main variables that affect the measurement of cholinesterases in pesticide poisoning studies. Methodology: A systematic review was performed in the PubMed, Crossreff and Google Scholar search engines, using the keywords cholinesterase, acetylcholinesterase, pesticides and intoxication, only original articles were taken, which measured cholinesterases by colorimetric and $\mathrm{pH}$ methods, with the objective of relating them to acute or chronic poisoning by pesticides. Results: $71.42 \%$ of the articles reported a decrease in cholinesterase in the population under study and $28.57 \%$ report not having detected a decrease in cholinesterase in the study population. Conclusions: It is recommended that researchers always consider the delicacy of these enzymes and remember the most important factors that can affect cholinesterase values in addition to pesticides.
\end{abstract}

Keywords:

cholinesterase, acetylcholinesterase, pesticides, poisoning

\begin{abstract}
Resumen:
La atención clínica de los intoxicados, así como las actividades de vigilancia epidemiológica de la población expuesta a plaguicidas demandan disponer de valores de referencia para poder tomar decisiones con base científica. Las colinesterasas son biomarcadores descubiertos a inicios del siglo XX que en la actualidad funcionan como auxiliares en el diagnóstico de intoxicaciones agudas y crónicas. Objetivo: Realizar una revisión sistemática que permita identificar las principales variables que afectan la medición de colinesterasas en los estudios de intoxicación por plaguicidas. Metodología: Se realizó una revisión sistemática en los buscadores PubMed, Crossreff y Google Scholar, mediante las palabras clave colinesterasa, acetilcolinesterasa, plaguicidas e intoxicación, se tomaron en cuenta solo artículos originales, que midieran las colinesterasas mediante métodos colorimétricos y de $\mathrm{pH}$, con el objetivo de relacionarlas con intoxicaciones agudas o crónicas por plaguicidas. Resultados: El 71.42\% de los artículos reportaron una disminución de las colinesterasas en la población bajo estudio y el $28.57 \%$ refiere no haber detectado disminución de las colinesterasas en la población en estudio. Conclusiones: Recomendamos a los investigadores tener en cuenta en todo momento la delicadeza de estas enzimas y recordar los factores más importantes que pueden afectar los valores de las colinestera sas a demás de los pla guicid as.
\end{abstract}

\section{Palabras Clave:}

colinesterasa, acetilcolinesterasa, plaguicidas, intoxicación

\section{INTRODUCTION}

Cholinesterases are a family of carboxy esterases, which are present from single-celled organisms to multicellular organisms such as plants, invertebrates and vertebrates in which they appear in embryonic development before synaptogenesis. ${ }^{1}$ In 1914, the neurotransmitter acetylcholine (ACh) was discovered which is essential for the central nervous system, the peripheral nodes and the neuromuscular plate. Acetylcholine binds to a specific receptor on the postsynaptic membrane; resulting in the change of membrane potential and may be the synapse. ${ }^{1}$ Subsequently, the acetylcholine will be hydrolyzed by acetylcholinesterase (AChE) becoming acetic acid and choline, through the postsynaptic membrane return to the state of rest. ${ }^{2}$ This process prevents overstimulation of muscle fibers due to accumulation of acetylcholine at the neuromuscular junction, which causes a

\footnotetext{
a Corresponding author, Sociedad Mexicana de Salud Púbica, https://orcid.org/0000-0003-0979-6079, Email: azucenabenitezm@ gmail.com b Universidad Autónoma de Guerrero. Unidad Académica De Ciencias Químico-Biológicas, https://orcid.org/0000-0001-8502-982X, Email: marvar@uagro.mx
} 
wide range of neurotoxic effects (muscarinic, nicotinic, and neurological). ${ }^{2,3}$ See Table 1.Cholinergic syndrome. The accumulation effects of acetylcholine can be evidenced biochemically by suppressed levels of serum pseudocholinesterase (BChE) and erythrocyte cholinesterase (AChE). ${ }^{4}$ These two types of cholinesterase are divided according to their substrate specificity, susceptibility to inhibitors, and energy tissues: Acetylcholinesterase (AChE), or true cholinesterase or E-type specific cholinesterase, is predominant in muscle, erythrocytes, and the nervous system, where BChE levels are lower. ${ }^{2}$

Butyrylcholinesterase (BChE) or pseudocholinesterase, plasma cholinesterase or cholinesterase type $\mathrm{S}$ is present in tissues such as intestine, kidney, heart, lungs and mainly the liver. After being synthesized, it is secreted into plasma. ${ }^{1,2}$ The physiological function of $\mathrm{BChE}$ is still unknown, it is speculated that it plays an important role in the metabolism of lipids and lipoproteins, regulating the concentration of choline in plasma or preventing the accumulation of butyrylcholine, during the metabolism of fatty acids and lipogenesis. In addition, they have detected functions in the metabolism of drugs such as procaine, succinylcholine and acetylsalicylic acid, as well as the elimination of phosphates and carbamates. ${ }^{5}$

Cholinesterase enzymes are frequently used to biomonitor exposure to organophosphates and carbamates, which are determined to be neurotoxic and cause $80 \%$ of acute or chronic pesticide poisonings world wide. ${ }^{6}$ Pesticide absorption pathways are by inhalation, ingestion and through intact skin. Once in the body they suffer metabolic reactions of activation, detoxification and conjugation. Its mechanism of toxic action is the binding and inhibition of stearic enzymes in a stable manner, and they are eliminated relatively quickly by the renal route ${ }^{3}$ The inhibition of AChE, caused by carbamates, is of less persistence since it is decarbamilated in a reversible and spontaneous way, with neurotoxic effects similar to organophosphates, but to a lesser extent and with usually rapid recovery. ${ }^{6}$ Approximately 3 million people suffer from poisoning and 200,000 die from pesticide poisoning annually in the world. ${ }^{2,7}$ Millions are exposed to hazardous work practices and insecure storage of pesticides; however, it is deliberate self-poisoning that causes most deaths, particularly in Asia. ${ }^{8}$

Table 1. Cholinergic syndrome.

\begin{tabular}{|l|l|}
\hline Receptors & Signs and symptoms \\
\hline Muscarinic & $\begin{array}{l}\text { Sialorrhea, tearing, urinary incontinence, diarrhea, } \\
\text { tenesmus, bronchospasm, bronchrhea, wheezing, } \\
\text { decreased blood pressure, bradycardia, nausea a, vomiting, } \\
\text { cramping, sweating, myosis and blurred vision. }\end{array}$ \\
\hline Nicotinic & $\begin{array}{l}\text { Muscle fatigue, muscle spasm, fasciculations, paralysis, } \\
\text { decreased respiratory effort, tachycardia, diaphoresis, } \\
\text { hypertension, hypoglycemia, paleness, cyanosis and } \\
\text { mydriasis. }\end{array}$ \\
\hline
\end{tabular}

\begin{tabular}{|l|l|}
\hline $\begin{array}{l}\text { Central } \\
\text { nervous } \\
\text { system }\end{array}$ & $\begin{array}{l}\text { Anxiety, nervousness, confusion, headache, seizures, } \\
\text { ataxia, bradilalia, emotional lability, Cheyne-Stokes }\end{array}$ \\
& $\begin{array}{l}\text { breathing, respiratory center. depression, central } \\
\text { respiratory paralysis, dyslexia,coma and insomnia. }\end{array}$ \\
\hline
\end{tabular}

Source: Adapted from Virú Loza MA. Current management of acute cholinesterase inhibitor poisoning: misconceptions and the need for updated Peruvian guidelines. An. Fac. med. 2015;76(4):431-7.

Chronic intoxications or exposures are associated with immunological effects, carcinogenic effects, reproductive disorders, developmental disorders, neurotoxicity, even exposure to pesticides is associated with the development of Parkinson's, Alzheimer's, anxiety disorders, deficit disorders. attention with hyperactivity, and depression, including areas with an intensive pesticide have a high suicide rate. ${ }^{9-11}$

\section{Cholinesterase levels as a diagnosis}

Every good diagnosis should start with a complete medical history, through the suspicion or certainty of exposure to the pesticide, the route of absorption and a compatible clinical picture. Diagnostic confirmation should ideally be done by measuring cholinesterase activity. ${ }^{4}$ However, the results should be interpreted with caution because a wide variability has been observed in the values considered normal because of the characteristics of cholinesterase, individual differences mainly the aggregate clinical conditions, substances that can alter cholinesterase levels, influence of the environment and the possibility of laboratory errors. ${ }^{7,11,12 .}$

\section{MATERIALS AND METHODS}

A systematic review was performed in the PubMed, Crossreff and Google Scholar search engines, using the keywords cholinesterase, acetylcholinesterase, pesticides and poisoning.

- Inclusion criteria: Only original articles in English or Spanish were taken into account, the search did not include a publication date limit, in their methodology they had to include the measurement of plasma cholinesterase, erythrocyte, or both through colorimetric chemical reactions or changes in $\mathrm{pH}$, who would have carried out their measurements in vivo, included experimental and non-experimental investigations.

- Exclusion criteria: Those with a sample of less than 15 people and original articles that did not have the objective of relating cholinesterase measurements with acute or chronic pesticide poisoning.

\section{PROCESS}

The review, synthesis and classification of the selected works was carried out by preparing a matrix that includes the name of the first author, date of publication, country of origin, methodology and type of cholinesterase measured, sample size, presence of signs of pesticide poisoning, number of people with inhibited or altered cholinesterase, if there is a statistical 
association with cholinesterase, exposure time and exclusion criteria. The table is sorted by dates from oldest to most current. See Table 2. Studies with measurements of cholinesterases and pesticide poisoning.

\section{RESULTS}

Twenty eight original articles that describe and analyze situations organophosphate or carbamate poisoning and its association with cholinesterase were used to generate this article. Twenty eight point five percent belong to PubMed, $35.7 \%$ to Crossreff and the remaining $35.7 \%$ is owned by Google Scholar.

Selected articles are conducted primarily in the Americas, predominantly Colombia with 8 items, with the exception of 5 items from which, 3 were conducted in India, 1 in Thailand and 1 in Serbia.

The analytical methods used were the Ellman method, the method of Ellman with DTNA (6-6'-ditiodinicotínico acid), Magnotti, Michel, Lovibond, Wiener, Merckotest, Aldridge and Randox which measure through chemical reactions or changes colored $\mathrm{pH}$. The method most commonly found in the articles as the method of Ellman.

Normal values vary widely considered in each study, according to the methodology, the laboratory, the sample type and population in which was measured for both plasma cholinesterase to erythrocyte cholinesterase. $13(46.42 \%)$ of the items evaluated plasma cholinesterase, $11(39.28 \%)$ evaluated. and 4 erythrocyte cholinesterase $(14.28 \%)$ of the items evaluated both. Only $10(35.71 \%)$ of the studies collected describe the methodology with which the sample was taken. See Figure 1. Sampling and exclusion criteria for cholinesterase.

The study with the lowest sample had 15 participants and the largest sample had 46.290 participants. 10 (35.71\%) of the articles included the exposed group and the control group in 4 (11.2\%) of the studies reported taking between 3 and 5 serial samples per person and one article made measurements cholinesterase saliva.

The $28(100 \%)$ articles described their population, as affected by pesticides, with risk factors and little or no protective equipment of these 20 articles $(71.4 \%)$ reported signs and symptoms of poisoning, of these 20 articles that reported signs and symptoms, 6 (30\%) focus on symptoms of chronic poisoning and $14(70 \%)$ articles described signs and symptoms of acute poisoning.

Twenty $(71.42 \%)$ of the articles reported a decrease in cholinesterase in the study population and 8 articles (28.57\%) reported not having detected a decrease in cholinesterase in the study population.

Of the 20 articles that reported a decrease in cholinesterase:
- $9(45 \%)$ articles indicated that less than $15 \%$ of their total population studied presented a decrease in measured cholinesterase enzymes.

- $5(25 \%)$ articles reported that between $16 \%$ to $50 \%$ of their total population had a decrease in measured cholinesterase enzymes.

- $6(30 \%)$ of the articles reported that between $51 \%$ to $100 \%$ of their study population selected a decrease in measured cholinesterase enzymes.

In $4(14.28 \%)$ articles, they found no statistical association between pesticide exposure and cholinesterase levels. In 9 $(32.14 \%)$ articles, the exposure time of their population to pesticides was not reported and in the remaining 19 (67.85\%) articles, the exposure time was reported between 6 hours to 50 years. Only $14(50 \%)$ of the studies used for this article mentioned or described their exclusion criteria when choosing their sample, ruling out physiological, pathological conditions or the presence of substances that can alter cholinesterase levels. For example, medications used in the treatment of Alzheimer's, such as donepezil or galantamine, have an inhibitory action against BuChE and AchE. ${ }^{13}$

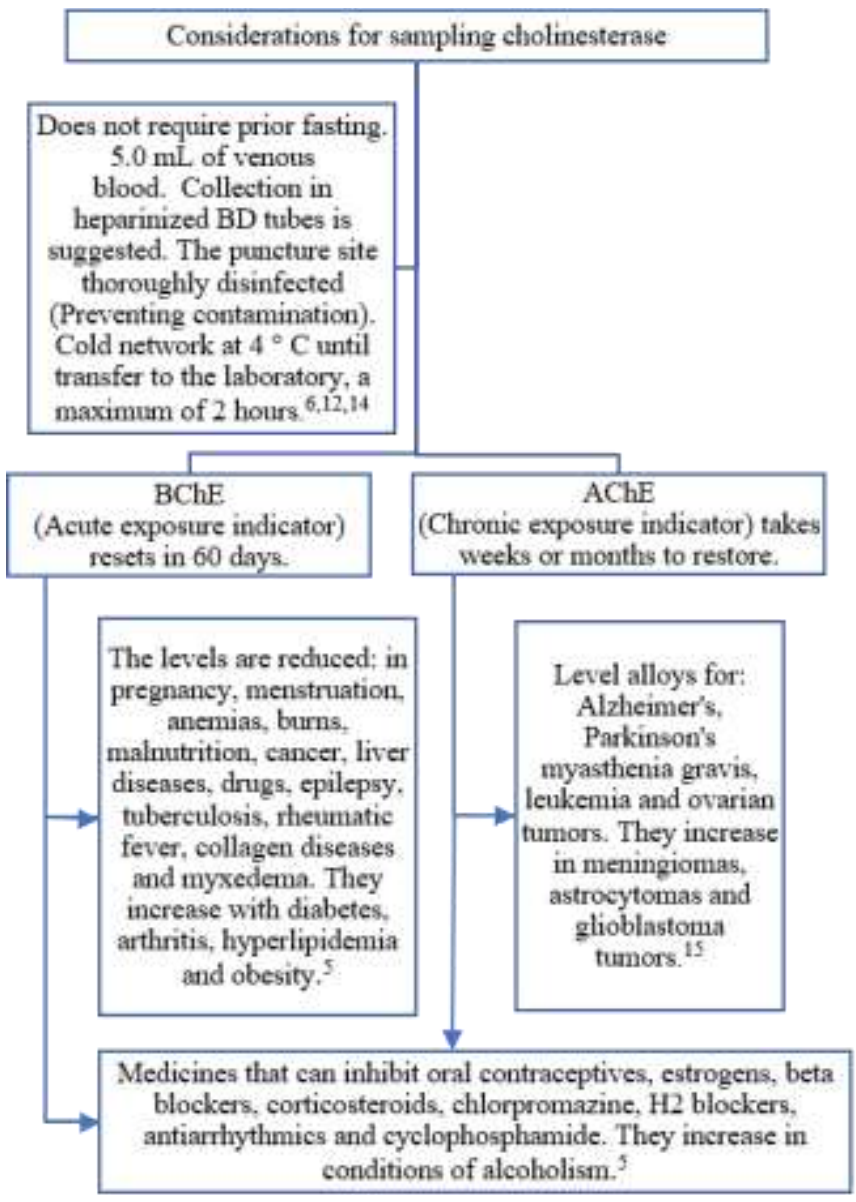

Figure 1 Sampling and exclusion criteria for cholinesterase. 
Biannual Publication, Mexican Journal of Medical Research ICSa, Vol. 9, No. 17 (2021) 47-55

Table 2. Studies with cholinesterase measurement and pesticide poisoning.

\begin{tabular}{|c|c|c|c|c|c|c|c|}
\hline Studies & Enzyme and method & Sample size & Signs and symptoms & $\begin{array}{c}\text { Cholinesterase } \\
\text { inhibited }\end{array}$ & Statistical association with cholinesterase & Exhibition time & $\begin{array}{c}\begin{array}{c}\text { Exclusion } \\
\text { criteria }\end{array} \\
\end{array}$ \\
\hline $\begin{array}{l}\text { Varona M. } \\
1998 \\
\text { Colombia. }{ }^{16}\end{array}$ & $\begin{array}{c}\text { AChE } \\
4 \text { methods: compare } 190 \\
\text { samples by Merckotest, } \\
\text { Michel in plasma, } \\
\text { Michel and Aldrige in } \\
\text { whole blood and } \\
\text { Lovibond. }\end{array}$ & $\begin{array}{c}\text { Between } 1993 \text { and } \\
1995, \text { a total } \\
\text { population of } 46,290 \\
\text { participated. }\end{array}$ & NA & $\begin{array}{c}\text { Of a total of } 46,290, \\
5,370 \text { tests were } \\
\text { below the normal } \\
\text { range, equivalent to } \\
11.6 \% \text {. }\end{array}$ & $\begin{array}{l}\text { The economic activities that presented } \\
\text { higher prevalence of abnormal values for } \\
\text { AChE, corresponded to the urban } \\
\text { application, formulator and aerialapplication }\end{array}$ & NA & NA \\
\hline $\begin{array}{l}\text { Luna-Avilés I } \\
2007 \\
\text { Nicaragua. }{ }^{17}\end{array}$ & $\begin{array}{c}\text { AChE } \\
\text { Lovibond Method } \\
\text { BRV: } 0.35-0.45 \mathrm{ml}\end{array}$ & $\begin{array}{c}60 \text { samples } \\
30 \mathrm{EX} \text { and } 30 \mathrm{NE}\end{array}$ & $\begin{array}{l}\text { Genetic damage in } \\
\text { micronuclei, premature } \\
\text { aging }\end{array}$ & $\begin{array}{c}6 \text { people of the } \\
\text { exposed }(20 \%) \text { and } \\
1 \text { of the non- } \\
\text { exposed }(3.3 \%)\end{array}$ & $\begin{array}{c}\text { AchE levels indicate chronic neurotoxic } \\
\text { effect in pesticide traders. Significant } \\
\text { difference between Control and Exposed } \\
\text { groups ( } \mathrm{z}=2.5 \text {, CI: } 99 \%)\end{array}$ & $\begin{array}{l}\text { Exposure range } \\
\text { from } 2 \text { to } 11 \\
\text { years }\end{array}$ & NA \\
\hline $\begin{array}{l}\text { Lamadrid-BoadaI } \\
\text { AI. } 2011 \\
\text { Cuba. }{ }^{18}\end{array}$ & $\begin{array}{c}\text { AChE } \\
\text { Method regulated by the } \\
\text { MINSAP. } \\
\text { ARV: } 0,3950 \pm 0,0275 \\
(0,340-0,450)\end{array}$ & $8 \mathrm{EX}$ and $15 \mathrm{NE}$. & $\mathrm{NA}$ & 0 & $\begin{array}{l}\text { BChE activity values, during the } 2 \text { years of } \\
\text { the study, remained within the reference } \\
\text { value for the Cuban population a verage. }\end{array}$ & $\begin{array}{c}\text { Average } \\
\text { working years: } \\
7.6\end{array}$ & $\begin{array}{c}\text { Complete } \\
* * *\end{array}$ \\
\hline $\begin{array}{c}\text { Gutiérrez- } \\
\text { Coronado ML. } \\
2012 \\
\text { Mexico. }^{19}\end{array}$ & $\begin{array}{c}\text { BChE } \\
\text { Colorimetric method } \\
\text { (Randox diagnostic kit) } \\
\text { BRV: } 7,68 \pm 2,81 \mathrm{kU} / \mathrm{L}\end{array}$ & 212 farmers & $\begin{array}{l}98.6 \% \text { have some type of } \\
\text { sign or symptom due to } \\
\text { poisoning. symptoms } \\
\text { more often headache and } \\
\text { fatigue. }\end{array}$ & $\begin{array}{l}1.8 \% \text { presented } \\
\text { values below the } \\
\text { reference values, } 1 \% \\
\text { women with low } \\
\text { values }\end{array}$ & No significant association was found. & $\begin{array}{c}35.3 \% \text { less than } \\
5 \text { years }\end{array}$ & NA \\
\hline $\begin{array}{l}\text { Uzcátegui-Nava J. } \\
2013 \text { Venezuela. }\end{array}$ & $\begin{array}{l}\text { AChE human saliva ** } \\
\text { Ellman Method }\end{array}$ & $\begin{array}{l}60 \text { saliva samples } \\
\text { (sputum) from } \\
\text { farmers. }\end{array}$ & $\mathrm{NA}$ & $\begin{array}{c}80 \% \text { enzyme } \\
\text { inhibition at } \\
\text { concentrations less } \\
\text { than } 100 \mathrm{ppm} \\
\text { pesticide. }\end{array}$ & $\begin{array}{l}\text { It was observed that orga nophosphorus } \\
\text { pesticides and carbamates significantly } \\
\text { affect the activity values of the enzyme } \\
\text { acetylcholinestera se in saliva. }\end{array}$ & NA & NA \\
\hline $\begin{array}{l}\text { Prasad D. } \\
2013 \\
\text { India. }^{8}\end{array}$ & $\begin{array}{c}\text { BChE** } \\
5 \text { sampling } \\
\text { BRV } 5100-11700 \mathrm{U} / \mathrm{L}\end{array}$ & $\begin{array}{l}76 \text { patients poisoned } \\
\text { with phosphorous } \\
\text { organ }\end{array}$ & $\begin{array}{c}\text { Congested conjunctiva } \\
87 \% \text {, pointed pupil } 83 \%, \\
\text { non-reactive pupil } 75 \%, \\
\text { difficulty, respiratory } \\
60 \% \text { and abdominal pain } \\
37 \% \text {. } \\
\end{array}$ & $\begin{array}{l}62 \text { patients }(90 \%) \\
\text { had BChE values of } \\
\text { less than } 5000 \mathrm{iu}\end{array}$ & $\begin{array}{l}\text { Respiratory distress and fasciculation were } \\
\text { related to }>75 \% \text { reduction in BChE. } \\
\text { constricted pupil and congested conjunctiva } \\
\text { a } 50-75 \% \text { reduction }\end{array}$ & NA & $\begin{array}{c}\text { Complete } \\
* * * *\end{array}$ \\
\hline $\begin{array}{c}\text { Chaudhary SC } \\
2013 \\
\text { India. }^{4}\end{array}$ & $\begin{array}{c}\text { BChE ** } \\
\begin{array}{l}\text { Spectrophotometry } \\
\text { method }\end{array} \\
\text { BRV: } 7,000-19,000 \text { ui / } 1 \\
\end{array}$ & $\begin{array}{l}70 \text { patients } \\
2 \text { groups }\end{array}$ & $\begin{array}{c}60 \% \text { required ventilatory } \\
\text { support from group B } \\
\text { and } 48.57 \% \text { from group } \\
\text { A } \\
\end{array}$ & $\begin{array}{l}\text { BChE levels were } \\
\text { reduced in all cases. } \\
100 \%\end{array}$ & $\begin{array}{c}\text { About half of the patients have mild } \\
\text { poisoning according to the classification of } \\
\text { Kumaret al. }\end{array}$ & $\begin{array}{l}6 \text { hours after } \\
\text { exposure. }\end{array}$ & $\begin{array}{c}\text { Complete } \\
* * *\end{array}$ \\
\hline $\begin{array}{c}\text { Ramirez-Santana } \\
\text { M. } \\
2015 \\
\text { Chile. }{ }^{20}\end{array}$ & $\begin{array}{l}\text { BChE and } \mathrm{AChE} * * \\
\text { the acylpeptide } \\
\text { hydrolase (ACPH) } \\
3 \text { samples exposed to } \\
3 \text { groups }\end{array}$ & $\begin{array}{c}100 \text { exposed } \\
100 \text { internal controls } \\
\text { and } \\
1000 \text { external } \\
\text { controls }\end{array}$ & $\begin{array}{c}\text { Memory, praxis } \\
\text { constructive, attention, } \\
\text { motorfunction and } \\
\text { executive. }\end{array}$ & $\mathrm{NA}$ & $\begin{array}{l}\text { These enzymes are inhibited when people } \\
\text { are exposed to high doses of } \\
\text { orga nophosphates, with clear signs and } \\
\text { symptoms of acute poisoning; therefore, they } \\
\text { do not serve to identify the risk of chronic } \\
\text { exposure. }\end{array}$ & $\begin{array}{c}\text { Minimum span } \\
5 \text { years }\end{array}$ & Complete \\
\hline $\begin{array}{c}\text { Lozano-Socarrás } \\
\text { SL. } \\
2015 \\
\text { Colombia. }^{21}\end{array}$ & $\begin{array}{c}\text { BChE } \\
\text { Wiener Method } \\
\text { Colorimetric } \\
\text { BRV 3200-9000 U/L }\end{array}$ & 80 farmers & $\overline{\mathrm{NA}}$ & 10 farmers $(12.5 \%)$ & $\begin{array}{c}\text { At higher exposure time, individuals } \\
\text { presented high levels of the enzyme }(\text { Rho }= \\
0.408 ; \mathrm{p}<0.05) .\end{array}$ & $\begin{array}{l}70,8 \pm 91,6 \\
\text { months }\end{array}$ & $\overline{\mathrm{NA}}$ \\
\hline
\end{tabular}


Biannual Publication, Mexican Journal of Medical Research ICSa, Vol. 9, No. 17 (2021) 47-55

\begin{tabular}{|c|c|c|c|c|c|c|c|}
\hline Studies & Enzyme and method & Sample size & Signs and symptoms & $\begin{array}{c}\text { Cholinesterase } \\
\text { inhibited }\end{array}$ & Statistical association with cholinesterase & Exhibition time & $\begin{array}{c}\text { Exclusion } \\
\text { criteria }\end{array}$ \\
\hline $\begin{array}{l}\text { Blanco AR } 2016 \\
\text { Honduras. }\end{array}$ & $\begin{array}{c}\text { AChE } \\
\text { Michel Method } \\
\text { 2 groups EX y NE } \\
\text { VRA: } 154 \text { unids de } \\
\text { pH/hour }\end{array}$ & $\begin{array}{l}95 \text { farmers, } 60 \mathrm{EX} \\
\text { and } 35 \mathrm{NE}\end{array}$ & $\begin{array}{l}42.5 \% \text { have headache, } \\
\text { nausea, dizziness, } \\
\text { muscle weakness, eye } \\
\text { and skin irritation }\end{array}$ & $\begin{array}{l}3(5 \%) \text { EX y } 5 \\
(14.28 \%) \mathrm{NE}\end{array}$ & $\begin{array}{l}\text { Slight inhibition } 133 \text { units of } \mathrm{pH} / \text { hour and } \\
\text { values less than } 120 \text { units of } \mathrm{pH} / \text { hour as } \\
\text { moderate inhibition. }\end{array}$ & $\begin{array}{l}50 \% \text { of the } \\
\text { farmers have } \\
\text { worked more } \\
\text { than } 10 \text { years. }\end{array}$ & NA \\
\hline $\begin{array}{l}\text { Farrukh J. } \\
2016 \\
\text { India. }{ }^{23}\end{array}$ & $\begin{array}{c}\text { BuChe } \\
\text { Maw the Accucare kit } \\
\text { method of Knedel et al. } \\
\text { (1967) }\end{array}$ & $\begin{array}{c}374 \text { workers } \\
187 \mathrm{EX} \text { and } 187 \mathrm{NE}\end{array}$ & $\begin{array}{l}\text { Anxiety, insomnia, } \\
\text { severe depression, } \\
\text { disorders that affect } \\
\text { psychomotor speed, } \\
\text { selective attention, } \\
\text { divided attention, verbal } \\
\text { memory, eczema, } \\
\text { fatigue, headache, } \\
\text { sweating, abdominal } \\
\text { pain, nausea, muscle } \\
\text { weakness }\end{array}$ & NA & $\begin{array}{c}\text { The rates of anxiety and insomnia }(\mathrm{p}= \\
0.030) \text { and severe depression }(\mathrm{p}<0.001) \\
\text { were significantly higher in } \\
\text { organophosphorus pesticide sprayers than in } \\
\text { controls. }\end{array}$ & $\begin{array}{l}\text { Years } 9.88 \pm \\
\quad 7.72\end{array}$ & Complete $* * *$ \\
\hline $\begin{array}{c}\text { Restrepo-Cortés } \\
\text { B. } \\
2017 \\
\text { Colombia. }^{5}\end{array}$ & $\begin{array}{c}\text { AChEy BChE** } \\
\text { Ellman with DTNA } \\
\text { BRV } 12120-2176 \mathrm{u} / 1 \\
\text { ARV } 10567-2227 \mathrm{u} / 1\end{array}$ & $\begin{array}{l}819 \text { farmers }(480 \\
\text { men and } 339 \\
\text { women). }\end{array}$ & NA & $\begin{array}{l}36 \% \text { had inhibition } \\
\text { of AChE activity } \\
\text { and } 51 \% \text { of BChE* }\end{array}$ & $\begin{array}{l}\text { AChE was significantly lower among } \\
\text { fumigants }(\mathrm{P}=0.022) .\end{array}$ & NA & $\underset{* * *}{\text { Complete }}$ \\
\hline $\begin{array}{c}\text { Toro-Osorio MB. } \\
2017 \\
\text { Colombia. }^{6}\end{array}$ & $\begin{array}{c}\text { BChE } * * \\
\text { Ellman. Method } \\
\text { BRV } 4659-14443 \mathrm{u} / 1\end{array}$ & 1098 farmers. & $\begin{array}{l}15 \% \text { headaches, } 12 \% \\
\text { dizziness and } 8 \% \\
\text { blurred vision and } \\
\text { muscular aches. }\end{array}$ & $3.8 \%$ of farmers & $\begin{array}{l}\text { Less time after the last application of the } \\
\text { insecticide, there is a greater inhibition of the } \\
\text { enzyme. }\end{array}$ & $\begin{array}{l}\text { Range of one to } \\
\text { ten days since } \\
\text { the last } \\
\text { application }\end{array}$ & $\mathrm{NA}$ \\
\hline $\begin{array}{l}\text { Cuaspud J. } \\
2017 \\
\text { Ecuador. } \\
24\end{array}$ & $\begin{array}{c}\text { AChE Ellman Method } \\
\text { with DTNA } \\
\text { ARV EX 4199-2320.5 } \\
\text { u/l } \\
\text { ARV NE } 4745-3081 \mathrm{u} / 1 \\
\text { AV NE: } 3625,41 \mathrm{u} / 1\end{array}$ & $\begin{array}{c}145 \text { samples } \\
95 \mathrm{EX} \text { and } 50 \mathrm{NE} \text {. }\end{array}$ & $\begin{array}{l}18 \text { people- neurological } \\
\text { symptoms, } 35 \text { people- } \\
\text { muscarinic symptoms } \\
\text { and } 43 \text { people-nicotinic } \\
\text { symptoms. }\end{array}$ & $42 \%$ of farmers & $\begin{array}{c}\mathrm{AChE}(3154,99 \mathrm{U} / \mathrm{L}) \\
\text { Below the average level of the unexposed } \\
\text { group. }\end{array}$ & NA & Complete \\
\hline $\begin{array}{c}\text { Matheus T. } \\
\text { 2017 Venezuela. }{ }^{25}\end{array}$ & $\begin{array}{c}\text { BChE } \\
\text { BRV 3528-1162,45u/l } \\
\text { and the presence of } \\
\text { micronuclei }\end{array}$ & $\begin{array}{l}82 \text { samples } 41 \mathrm{EX} \\
\text { and } 41 \mathrm{NE} \text {. }\end{array}$ & $\begin{array}{l}\text { Prevalence of eye } \\
\text { redness } 48.8 \% \text { and } \\
\text { headache } 36.6 \%\end{array}$ & NA & $\begin{array}{c}\text { BChE activity in EX with statistically } \\
\text { significant differences, a negative correlation } \\
\text { of exposure time wa s observed. }\end{array}$ & $\begin{array}{l}\text { Working age of } \\
\text { at least } 6 \\
\text { months. } \\
\text { the range of } 6 \text { - } \\
15 \text { years }\end{array}$ & $\begin{array}{c}\text { NA } \\
\text { (exclusion } \\
\text { criteria only } \\
\text { for } \\
\text { micronuclei) }\end{array}$ \\
\hline $\begin{array}{l}\text { Butinof M. } 2017 \\
\text { Argentina. }^{14}\end{array}$ & $\begin{array}{c}\text { BChE } \\
\text { BRV: } 3200 \text { a } 9000 \mathrm{u} / \mathrm{l}\end{array}$ & 47 farmers & $\begin{array}{c}\text { Breast cancer and total } \\
\text { cancer }\end{array}$ & NA & $\begin{array}{l}\text { The cumulative exposure to pesticides and } \\
\text { BChe activity were negatively correlated }\end{array}$ & NA & $\begin{array}{c}\text { Complete } \\
* * *\end{array}$ \\
\hline $\begin{array}{l}\text { Díaz SM. } \\
2017 \text { Colombia. }\end{array}$ & $\begin{array}{l}\text { BChE y AChE** } \\
\text { Michel method. }\end{array}$ & 125 farmers & $\begin{array}{l}\text { Eye burning with } 72.0 \% \\
\text { (90), headache with } \\
68.0 \% \text { (85) and dizziness } \\
\text { with } 56.0 \% \text {. }\end{array}$ & $\begin{array}{l}8.0 \%(\mathrm{n}=10) \text { of } \\
\text { individuals have } \\
\text { AChE inhibition }\end{array}$ & $\begin{array}{l}\text { AChE inhibition was minimal, the use of } \\
\text { organophosphorus pesticides, carba mates, } \\
\text { a mong others, was reported. } \\
\text { There is no difference between age and } \\
\text { gender }\end{array}$ & $\begin{array}{l}\text { Range } 9 \text { months } \\
\text { to } 50 \text { years. } \\
\text { Minimum } \\
\text { previous } \\
\text { exposure } 5 \\
\text { days. }\end{array}$ & NA \\
\hline $\begin{array}{l}\text { Pedrozo ME, } \\
2017 \\
\text { Paraguay. }\end{array}$ & BChE & $15 \mathrm{EX}$ & $\begin{array}{l}\text { Nausea and vomiting, } \\
\text { followed by abdominal } \\
\text { pain, headache, fever, } \\
\text { pruritus, conjunctival } \\
\text { hyperemia and sweating }\end{array}$ & 0 & $\begin{array}{l}\text { Samples were taken in order to establish the } \\
\text { renal and hepatic profile, as well as serum } \\
\text { cholinesterase, with results within the } \\
\text { reference values. }\end{array}$ & $\begin{array}{l}48 \text { hours prior } \\
\text { exposure }\end{array}$ & Complete \\
\hline
\end{tabular}


Biannual Publication, Mexican Journal of Medical Research ICSa, Vol. 9, No. 17 (2021) 47-55

\begin{tabular}{|c|c|c|c|c|c|c|c|}
\hline Studies & Enzyme and method & Sample size & Signs and symptoms & $\begin{array}{c}\text { Cholinesterase } \\
\text { inhibited }\end{array}$ & Statistical association with cholinesterase & Exhibition time & $\begin{array}{c}\text { Exclusion } \\
\text { criteria }\end{array}$ \\
\hline $\begin{array}{l}\text { Lutovac M. } \\
2017 \\
\text { Servia. }^{28}\end{array}$ & $\begin{array}{c}\text { AChE } \\
6 \text { groups, } 4 \text { EX and } 2 \\
\text { NE. } \\
\text { A kinetic method with } \\
\text { propionaldehyde as } \\
\text { substrate and DTNB } \\
2618-6971 \mathrm{U} / \mathrm{L} \\
1 \text { shows per year }\end{array}$ & 175 workers & $\begin{array}{l}\text { Nervous disorders, } \\
\text { headache, dizziness, } \\
\text { paralysis, anxiety, } \\
\text { stomach pain, vomiting, } \\
\text { diarrhoea, a sphyxia or } \\
\text { increased sweating, } \\
\text { salivation, tears. }\end{array}$ & $\begin{array}{l}72 \%, \text { there is a } \\
\text { slight drop of AChE } \\
\text { activity each year. }\end{array}$ & $\begin{array}{l}\text { In workers who are constantly exposed to } \\
\text { pesticides, acetylcholinestera se is within the } \\
\text { reference value. It observed in } 72 \% \text { a slight } \\
\text { drop of AChE activity each year. }\end{array}$ & $\begin{array}{c}\text { Range 1-13 } \\
\text { years. }\end{array}$ & Complete \\
\hline $\begin{array}{l}\text { Guytingcoa. } \\
2017 \\
\text { Thailand. } \\
{ }^{29}\end{array}$ & $\begin{array}{c}\text { AChE ** } \\
\text { colorimetric method } \\
\text { below } 75 \text { units / ml }\end{array}$ & $\begin{array}{c}3,431 \text { workers gave } \\
\text { blood samples }\end{array}$ & $\begin{array}{c}\text { The most common self- } \\
\text { reported symptoms were } \\
\text { cough }(5.7 \%) \text {, fatigue } \\
(5.4 \%) \text {, dizziness }(5.1 \%) \text {, } \\
\text { dry skin and irritation } \\
(4.8 \%) .\end{array}$ & $\begin{array}{l}\text { The prevalence of } \\
\text { abnormal results of } \\
\text { AChE was } 12.5 \% \text {. }\end{array}$ & $\begin{array}{c}\text { Male a gricultural workers had a significantly } \\
\text { higher risk than women farmers after } \\
\text { adjusting for sex and level of risk (OR = } \\
1.56, \mathrm{P}<0.05) \text {. }\end{array}$ & $\mathrm{NA}$ & Complete \\
\hline $\begin{array}{c}\text { Lindao-Córdova } \\
\text { VA. } \\
2018 \\
\text { Ecuador. }^{11}\end{array}$ & $\begin{array}{c}\text { BChE } \\
\text { The kinetic } \\
\text { spectrophotometric } \\
\text { method (405 nm at } 37^{\circ} \\
\text { C). (Kruskal Wallis) } \\
\end{array}$ & 170 farmers & NA & NA & $\begin{array}{l}\text { The relative humidity is directly proportional } \\
\text { to the cholinesterase level, however with the } \\
\text { temperature it has an inverse relationship }\end{array}$ & $\begin{array}{c}\text { Range of } 1 \text { to } \\
10 \text { years }\end{array}$ & NA \\
\hline $\begin{array}{c}\text { Marrero S. } \\
\text { 2018 Venezuela. }{ }^{30}\end{array}$ & $\begin{array}{c}\mathrm{BChE} \\
4,970-13,977 \mathrm{U} / \mathrm{L}\end{array}$ & $20 \mathrm{EX}$ and $10 \mathrm{NE}$ & $\begin{array}{l}30 \% \text { allergies and } 30 \% \\
\text { itching, statistically } \\
\text { significant compared to } \\
\text { those not exposed }(\mathrm{Z}= \\
2.21 ; \mathrm{p}=0.01)\end{array}$ & $\begin{array}{l}3(15 \%) \text { workers } \\
\text { had BChE } \\
\text { inhibition. }\end{array}$ & $\begin{array}{l}\text { Three exposed workers are below the range } \\
\text { normal, this being statistically significant } \\
\qquad(\mathrm{p}=0.005) .\end{array}$ & NA & Complete \\
\hline $\begin{array}{c}\text { Caro-Gamboa LJ. } \\
2019 \\
\text { Colombia. }\end{array}$ & $\begin{array}{c}\text { BChE } \\
\text { Ellman. Method } \\
\text { BRV: } 8063,78 \pm 1934.01 \\
\text { u/l }\end{array}$ & 76 farmers & NA & $\begin{array}{c}1.32 \%(1 / 76) \mathrm{had} \\
\text { values below } 3930 \mathrm{u} \\
/ 1 .\end{array}$ & $\begin{array}{l}\text { A decrease was observed that did not show } \\
\text { significant differences during the three } \\
\text { annualmeasurement periods }(\mathrm{p}=0.25) \text {. }\end{array}$ & $\begin{array}{l}\text { At least six } \\
\text { months prior to } \\
\text { the study }\end{array}$ & NA \\
\hline $\begin{array}{l}\text { Luna-Rondón JM. } \\
2019 \\
\text { Colombia. }^{32}\end{array}$ & $\begin{array}{c}\mathrm{AChE}^{* *} \\
4 \text { measurements }\end{array}$ & $\begin{array}{l}256 \text { fumigators, a } \\
\text { control group was } \\
\text { also included. }\end{array}$ & $\begin{array}{l}37.2 \% \text { with symptoms } \\
\text { for chronic intoxication. } \\
\text { Affective responses such } \\
\text { as depression, reluctance } \\
\text { that may be due to other } \\
\text { endogenous or } \\
\text { exogenous causes. }\end{array}$ & $\begin{array}{c}\text { The a verage of the } 4 \\
\text { measurements } \\
\text { ranges between } 33.8 \\
\text { and } 27.6 \mathrm{U} / \mathrm{g} \text { of } \\
\text { oxyhemoglobin, } \\
\text { with progressive } \\
\text { decreases in the } \\
\text { initial value (67\%) } \\
\text { in the 3rd and 4th } \\
\text { measurements. }\end{array}$ & $\begin{array}{l}\text { The AChE figures in non-fumigators } \\
\text { remained in the normal range and the values } \\
\text { of the fumigators showed fluctuations } \\
\text { outside the normal range, being interpreted } \\
\text { as the presence of affectation or inhibition. }\end{array}$ & 8 to 28 years & Complete $* * *$ \\
\hline $\begin{array}{l}\text { Polanco-López- } \\
\text { de-Mesa. } \\
\text { 2019 Colombia. }{ }^{33}\end{array}$ & AChE & $\begin{array}{c}40 \text { farmers } \\
21 \mathrm{NE} \text { and } 19 \mathrm{EX}\end{array}$ & $\begin{array}{c}\text { No symptoms associated } \\
\text { with pesticide poisoning } \\
\text { were found. }\end{array}$ & NA & $\begin{array}{l}\text { According to the levels of erythrocyte } \\
\text { cholinesterase in individuals who use or not } \\
\text { pesticides, the results showed a significant } \\
\text { value in those who do not use them ( } \mathrm{p}= \\
0.042)\end{array}$ & NA & NA \\
\hline
\end{tabular}


Biannual Publication, Mexican Journal of Medical Research ICSa, Vol. 9, No. 17 (2021) 47-55

\begin{tabular}{|c|c|c|c|c|c|c|c|}
\hline $\begin{array}{l}\text { Studies } \\
\end{array}$ & Enzyme and method & Sample size & Signs and symptoms & $\begin{array}{c}\text { Cholinesterase } \\
\text { inhibited }\end{array}$ & Statistical association with cholinesterase & Exhibition time & $\begin{array}{c}\text { Exclusion } \\
\text { criteria }\end{array}$ \\
\hline $\begin{array}{c}\text { Serrano-Medina } \\
\text { A. } \\
2019 \\
\text { México. }^{10}\end{array}$ & $\begin{array}{c}\text { AChE** } \\
\text { Ellman modified } \\
\text { colorimetric (worek } \\
\text { method). } \\
\text { Samples processed } \\
\text { immediately. }\end{array}$ & $\begin{array}{c}140 \text { farmers } \\
2 \text { groups EX and NE }\end{array}$ & $\begin{array}{c}31.4 \% \text { have a diagnosis } \\
\text { of major depression, } \\
\text { numbness }(51.4 \%), \\
\text { dizziness }(51.4 \%), \\
\text { abdominaldiscomfort } \\
(40.7 \%), \text { dyspnea } \\
(31.4 \%), \text { nocturia } \\
(34.3 \%) \text { and insomnia } \\
(25.7 \%) . \\
\end{array}$ & $\begin{array}{c}44 \text { people with } \\
\text { suicidal risk and } \\
\text { AChE inhibition. } \\
\text { High risk } 27 \\
(63.36 \%) \\
\text { Moderate Risk } 9 \\
(20.45 \%) \\
\text { Low risk } 8 \\
(18.18 \%) . \\
\end{array}$ & $\begin{array}{l}\text { The mean value of AChE enzyme activity } \\
\text { for the exposed population was significantly } \\
\text { lower }(178.88 \mathrm{mE} / \mathrm{min} \pm 37.48) \text { than the } \\
\text { enzyme activity of the unexposed group but } \\
\text { the difference was not statistically significant } \\
\qquad(\mathrm{t}(33)=0.677, \mathrm{p}=0.503)\end{array}$ & $\begin{array}{c}2.96 \text { years } \\
\text { (between } 0.5 \\
\text { and } 20 \text { years). }\end{array}$ & NA \\
\hline $\begin{array}{c}\text { Magalhães AFA. } \\
2019 \\
\text { México. }{ }^{34}\end{array}$ & $\begin{array}{c}\text { BChe } \\
\text { Maw DiaSys commercial } \\
\text { kit (Diagnostic Systems } \\
\text { GmbH) } \\
\text { VRB women: } 3930 \text { to } \\
10800 \text { U / L) and for } \\
\text { men }(4620 \text { to } 11500 \text { U / } \\
\text { L). } \\
\end{array}$ & 382 workers & $\begin{array}{l}5 \text { to } 30 \% \text { have headache, } \\
\text { blurred vision, pruritus, } \\
\text { nausea, dyspnea, dry } \\
\text { cough, watery eyes and } \\
\text { ocular hyperemia. }\end{array}$ & $\begin{array}{l}68 \% \text { show decrease } \\
\text { in BuChe activity. }\end{array}$ & $\begin{array}{c}\text { The majority }(68 \%) \text { presented decreased } \\
\text { BuChE activity, mainly farmers }(85.9 \%) \text {; } \\
57.3 \% \text { of workers were considered } \\
\text { intoxicated, } 61.6 \% \text { by agrochemicals and } \\
37.9 \% \text { by industrial chemicals. }\end{array}$ & $\begin{array}{l}55.2 \% \text { had up to } \\
9 \text { years of } \\
\text { activity }\end{array}$ & NA \\
\hline $\begin{array}{l}\text { Dutta S. } \\
2019 \\
\text { India. }^{35}\end{array}$ & $\begin{array}{l}\text { AChE and BChE } \\
\text { Ellman method } \\
4 \text { groups } \\
\text { EE, NE and consumers } \\
\text { of alcohol and snuff }\end{array}$ & 225 Individuals & $\begin{array}{c}\text { Like headache, } \\
\text { abdominalpain, nausea, } \\
\text { vomiting and watery } \\
\text { eyes }\end{array}$ & $\begin{array}{l}\text { Exposed }(\mathrm{N}=95 \text { is } \\
42.2 \%) \mathrm{SD} \pm 1.85 \\
6.43 \mathrm{AChE} \mathrm{BuChE} \\
\text { and SD } 3.50 \pm 1.89\end{array}$ & $\begin{array}{l}\text { AChE activity was half in individuals } \\
\text { exposed to pesticides than controls }(\mathrm{p} \leq \\
0.001) \text {. BuChE activity also decreased } \\
\text { significantly in individuals exposed to } \\
\text { pesticides than in controls }(\mathrm{p} \leq 0.001\end{array}$ & $\begin{array}{l}\text { Working range } \\
1 \text { to } 30 \text { years }\end{array}$ & Complete \\
\hline
\end{tabular}

NA: Not applicable, no data are mentioned in the study.

DTNA: 6-6'-dithiodinicotinic acid

MINSAP: The Ministry of Public Health of the Republic of Cuba

BRV: BChE reference values, ARV: AChE reference values y AV: average value

EX: Exposed to organophosphorus and carbamates. NE: Not exposed

* Taking as reference the 90th percentile, a $25 \%$ decrease in the values of cholinesterase activity.

** It refers to methodological characteristics of the sampling.

*** Excludes participants for physiological, pathological conditions or presence of substances that can skew the results. 


\section{DISCUSSION}

Cholinesterase enzymes are widely used as biological markers to detect pesticide poisoning, but the variability of the range considered "normal" prevents comparison between the results in different studies. As already mentioned, the range of normality can vary from individual to individual, from population to population, by pathologies, substances, the environment for example high temperature and humidity less than $40 \%$ favor skin absorption by decreasing cholinesterase levels and even by the laboratory and the method used for its analysis. It is true that there is no standardized and universally accepted laboratory technique or method for these types of studies, however, since 1961 international organizations have recommended the Ellman method, which is reflected in its predominance in the analyzed articles. ${ }^{12,36,37,38}$ It is desired to highlight some of the main errors that were detected, for which the results of the measurements with acetylcholinesterase are not significant, the lack of standardized analytical and preanalytical parameters can have a great influence on the variability of results since there are modifications to the conventional method there are laboratories that prepare their own reagents, while others use commercial kits. ${ }^{34}$ It is important to select whether to investigate acute or chronic intoxications to identify the characteristic signs and symptoms, while choosing the most appropriate type of cholinesterase with our study. Butyrylcholinesterase is recommended for acute intoxications and acetylcholinesterase for chronic intoxications. ${ }^{6}$ At the time of selecting the sample, in most of the studies there are no mentioned exclusion criteria aimed at ruling out factors of the individual that could alter the values considered as "normal", this reason may explain why in some studies they reported cholinesterase inhibition within the control group. The methodology in the sampling is referred to in less than $50 \%$ of the studies, but it is of vital importance, considering that it can be contaminated from the venous puncture (by pesticides present in the skin) and by requiring special conditions for its transport (network from cold to approximately 4 degrees Celsius for a maximum of 2 hours) that prevent the inactivation of enzymes during transport. It has been reported that the activity of the AchE in the laboratory at -20 degrees Celsius is stable during the first 7 days, subsequently its activity slowly decreases, it is $91.8 \%$ at 34 days, while the activity of $\mathrm{BChE}$ in plasma remained unchanged for 34 days. Only one of the studies reported that after the arrival of the laboratory it took 10 hours to perform the analysis and another study reported the analysis immediately. ${ }^{6,10,12,14}$ It is believed that better results are obtained in studies where the baseline cholinesterase levels of everyone can be taken prior to exposure to pesticides, but it is known that this is not always possible, it is recommended the use of control groups that function as regional baseline samples. Good results are also seen in studies where they take serial samples and compare them to measure enzyme inhibition.
An investigation was added to this study where the measurement of $\mathrm{AChE}$ and $\mathrm{BChE}$ in saliva is carried out, which is an interesting proposal for the silver problem in some studies that showed the loss of population, who refused to take venous blood because it was an invasive technique; however, it is considered that more research is required for the validation and standardization of this method. ${ }^{3}$ For the analysis of cholinesterase levels, it was found interesting the proposals where the level of intoxication was associated, with cholinesterase levels on a percentage scale. Example. "According to serum PCE levels, the severity of the poisoning was defined according to the scale of Kumary col."4

- Latent: BChE level> 50\% of normal or> 3,500 IU / L.

- Mild poisoning: BChE level $20-50 \%$ of normal or $>1,401$ 3,500 IU / L

- Moderate poisoning: BChE level 10-20\% of normal or 701$1,400 \mathrm{IU} / \mathrm{L}$

- Severe poisoning: the BChE level is $<10 \%$ of normal or $<700$ IU / L.4

And the Proudfoot classification, where mild organophosphorus toxicity is defined as a $\mathrm{BChE}$ reduction of less than $10 \%$ (fatigue, headache, nausea and vomiting), moderate toxicity as a $10-50 \%$ reduction (myosis, weakness general, dysarthria, fasciculation) and severe toxicity such as> $50 \%$ of the reduction (general fasciculation, flaccid paralysis, respiratory distress, loss of consciousness). 8

\section{CONCLUSION}

According to what it was found, it is recommended that researchers who carry out studies with cholinesterase enzymes do not lose sight of the delicacy of these enzymes in order to have the best possible results, not necessarily seek that the results are below a range established by the laboratory and take into account notes that the appearance of symptoms of intoxication depends more on the rate of cholinesterase inhibition than on the absolute level of activity found.32

\section{CONFLICT OF INTERESTS}

The authors declare that they have no conflict of interest.

\section{REFERENCES}

1. Sánchez-Chávez G, Salceda R. Enzimas polifuncionales: el caso de la acetilcolinesterasa. REB. 2008;27(2):44-51.

2. Sanchez LH, Medina OM, Gómez G, González CI, Flores-Vargas Ó. Laboratory genetic-based reference values for cholinestera se activity in a Colombian population: A step forward in personalized diagnostics. Biomedica. 2015;35(2):20-9.

3. Uzcátegui Nava J, González Carrero S, Zambrano Vergara R, Pereira Colls A. Validación de un método ana lítico para determinar la enzima acetilcolinesterasa (AChE) en saliva humana de poblaciones expuestas a plaguicidas organofosforados y carbamatos. Rev. Od. Los Andes. 2013;8(2):6-16.

4. Chaudhary SC, Singh K, Sawlani KK, Jain N, Vaish AK, Atam V, et al. Prognostic significance of estimation of pseudocholinesterase activity and role of pralidoxime therapy in organophosphorous poisoning. Toxicol. Int. 2013;20(3):214-7. 
5. Restrepo Cortés B, Londoño Franco AL, Sánchez López JF. Valores de colinestera sa plasmática y eritrocitaria con ácido6-6'-ditiodinicotínico (DTNA) como indicador. Rev. Colomb. Quim. 2017;46(1):13-9.

6. Toro-Osorio MB, Rojas-Rodríguez AE, Díaz-Zapata JA. Niveles de colinesterasa sérica en caficultores del Departamento de Caldas, Colombia.Rev. Salud Pública. 2017;19(3):318-24.

7. Virú Loza MA. Manejo actual de las intoxicaciones agudas por inhibidores de la colinestera sa: conceptos erróneos y necesidad de guías peruanas actualizadas. An. Fac. med. 2015;76(4):431-7.

8. Prasad RMMD, Jirli PS, Mahadevaiah M, Shivanagappa M. Relevance of plasma cholinesterase to clinical findings in acute organophosphorous poisoning. Asia Pac. J. Med. Toxicol. 2013;2(1):23-7.

9. Simoniello MF, Kleinsorge EC, Carballo MA. Evaluación bioquímica de trabajadores rurales expuestos a pesticidas. Medicina (B. Aires). 2010;70(6):489-98.

10. Serrano-Medina A, Ugalde-Lizárraga A, Bojorquez-Cuevas MS, Garnica-Ruiz J, González-Corral MA, García-Ledezma A, et al Neuropsychiatric Disorders in Farmers Associated with Organophosphorus Pesticide Exposure in a Rural Village of Northwest México. Int. J. Environ. Res. Public Healt. 2019 ;16(5):pii:E689.

11. Fernández DG, Mancipe LC, Fernández DC. Intoxicación por organofosforados. Rev. Fac. Med. 2010;18(1):84-92.

12. Lindao-Córdova VA, Jave-Nakayo JL, Retuerto-Figueroa MG, RamosSevilla EI, Jinez-Llangari PA. Efecto de la humedad relativa y la temperatura en la aplicación de insecticidas organofosforados y carbamatos e impacto en la colinesterasa de agro productores de tomate (Solanum Lycopersicum L.) en la localidad de "San Luis, Chimborazo, Ecuador. Eur. Sci. J. 2018;14(24):204-15.

13. Mozaffarnia S, Teimuri-Mofrad R, Rashidi MR. Design, synthesis and biological evaluation of 2,3-dihydro-5,6-dimethoxy-1H-inden-1-one and piperazinium salt hybrid derivatives as $\mathrm{hAChE}$ and $\mathrm{hBuChE}$ enzyme inhibitors. Eur. J. Med. Chem. 2020;191: pii: E112140.

14. Butinof M, Fernández R, Muñoz S, Lerda D, Blanco M, Lantieri JM, et al. Valoración de la exposición a plaguicidas en cultivos extensivos de Argentina y su potencialimpacto sobre la salud. Rev. Argent. Salud Pública. 2017;8(33):8-15.

15.Sánchez-Chávez G, Salceda R. Enzimas polifuncionales: el caso de la acetilcolinestera sa. REB. 2008;27(2):44-51

16.Varona M, Morales L, Ortiz J, Sánchez JF, Cárdenas O, Hoz F. Panorama epidemiológico de exposición a plaguicidas inhibidores de colinesterasa en 17 departamentos del país. Biomedica. 1998;18(1):229.

17. Luna-Avilés I, Gminski R, Mersch-Sundermann V, Huete-Pérez JA Determinación de daño genético en comerciantes de plaguicidas en el departamento de Matagalpa. Encuentro. 2007;(78):76-91.

18. Lamadrid BoadaI AI, Romero Aguilera I, González Mesa JE, Mandina Cardoso T. Biomonitoreo de trabajadores expuestos a plaguicidas. Rev. cuba. investig. bioméd. 2011;30(2):235-244.

19. Gutiérrez Coronado ML, Valenzuela Quintanar AI, Aldana Madrid ML, Grajeda Cota 1 P, Cabrera Pacheco RM, Ballesteros Vázquez MN, et al. Colinesterasa y paraoxonasa séricas como biomarcadores de exposición a plaguicidas en jornaleros agrícolas. Biotecnia. 2012; XIV(2):40-6

20. Ramírez-Santana M, Zúñiga L, Corral S, Sandoval R, Scheepers PT, Van der Velden K, et al. Assessing biomarkers and neuropsychological outcomes in rural populations exposed to organophosphate pesticides in Chile - study design and protocol. BMC Public Health 2015;15(116):1-9.

21. Lozano-Socarrás SL. Determinación del nivel de colinesterasa sérica en una población ocupacionalmente expuesta a plaguicidas en el municipio Zona Bananera, Magdalena (Colombia) 2012. Curare. $2015 ; 2(1): 23-8$
22. Blanco AR, Ponce HD, Lanza NA, Velásquez HJ, Calderón GL. Actividad de la colinesterasa total en pobladores que utilizan plaguicidas en La Brea, Lepaterique durante el año 2015. Rev. cienc. tecnol. 2016;(19):94-110.

23. Jamal F, Haque QS, Singh S. Interrelation of Glycemic Status and Neuropsychiatric Disturbances in Farmers with Organophosphorus Pesticide Toxicity. Open Biochem. J. 2016; 10:27-34.

24. Cuaspud J, Vargas B. Determinación de Colinesterasa Eritrocitaria en Trabajadores Agrícolas Expuestos a Plaguicidas Organofosforados y Carbamatos. Quim. Cent. 2017;1(1):71-82.

25. Matheus T, Aular Y, Bolaños A, Fernández Y, Barrios E, Hung ML. Actividad de butirilcolinesterasa y micronúcleos en trabajadores agrícolas con mezclas de plaguicidas. Salud Trab. 2017;25(1): 23-36.

26. Díaz SM, Sánchez F, Varona M, Eljach V, Muñoz N. Niveles de colinesterasa en cultivadores de papa expuestos ocupacionalmente a plaguicidas, Totoró, Cauca. Rev. Univ. Ind. Santander Salud. 2017;49(1):85-92

27. Pedrozo ME, Ocampos S, Galeano R, Ojeda A, Cabello A, Assis D. Casos de intoxicación aguda por plaguicidas en la colonia Puerto Pira pó, Ita púa, Para guay, febrero de 2014. Biomedica. 2017;37(2):158163.

28. Lutovac M, Popova OV, Jovanovic Z, Berisa H, Kristina R, Ketin S, Bojic M. Management, Diagnostic and Prognostic Significance of Acetylcholinesterase as a Biomarker of the Toxic Effects of Pesticides in People Occupationally Exposed. Open Access Maced. J. Med. Sci. 2017;5(7):1021-27

29. Guytingco A, Thepaksorn P, Neitzel RL. Prevalence of Abnormal Serum Cholinesterase and Associated Symptoms from Pesticide Exposure among Agricultural Workers in the South of Thailand J. Agromedicine. 2018;23(3):270-78.

30. Marrero S, Guevara H, Eblen-Zajjur A, Sequera M. Evaluación de la actividad de la colinesterasa, medio ambiente y geolocalización de trabajadores expuestos en una comunidad agraria de la Colonia Tovar, Venezuela. Rev. Latinoam. Patol. Clin. Med. Lab. 2018;65(1):45-54.

31. Caro-Ga mboa LJ, Forero-Castro RM,Torres-Torres VE, Suárez-Pulido DX, Dallos-Báez AE. Evaluación de colinesterasa plasmática en floricultores de los municipios de Chiquinquirá y Toca, Boyacá, Colombia.Pensam. Acción. 2019;(27):21-42.

32. Luna Rondón JM, Hanna La valle MI, Amador CE. Condición clínica y niveles de colinesterasa de trabajadores informales dedicados a la fumigación con plaguicidas. NOVA. 2019;17(31):67-77.

33. López-de-Mesa PY, Hernández-Carmona D, Escobar-Pérez ML, Aguirre-Acevedo DC, Parra-Hernández Á. Medición de niveles de colinestera sas eritrocitarias en agricultores usuarios de pla guicidas y en practicantes de a groecología, San Cristóbal, Medellín, Colombia. Rev. Fac. Nac. Salud Pública. 2019;37(3):25-33.

34. Maga lhães AFA, Caldas ED. Occupationalexposure and poisoning by chemical products in the Federal District. Rev. Bras. Enferm. 2019;72(1):32-40

35. Dutta S, BahadurM. Effect of pesticide exposure on the cholinesterase activity of the occupationally exposed tea garden workers of northern part of West Bengal, India. Biomarkers. 2019;24(4):317-24.

36. Carmona-Fonseca J. Valores de referencia de colinesterasa plasmática con los métodos de Michel, EQM ${ }^{\circledR}$ y Monotest ${ }^{\circledR}$ en población laboral activa del departamento de Antioquia, Colombia. Biomedica. 2003:23(4):437-55

37. Carmona-Fonseca J. Correlación y conversión entre los valores de actividad de la colinesterasa plasmática medida por tres técnicas diferentes. Rev. Panam. Salud Pública. 2007;22(1):35-40.

38. Bernal-Hernández YY, Aguilera-Márquez D, Grajeda-Cota P, ToledoIbarra GA, Moreno-Godínez ME, Perera-Ríos JH, et al. Actividad acetilcolinesterasa (ache) y butirilcolinesterasa (buche) en poblaciones mexicanas: estudio piloto. Rev. Int. Contam. Ambie. 2018;34:25-32. 